\title{
Epigenetic (de)regulation of adult hippocampal neurogenesis: implications for depression
}

\author{
António Mateus-Pinheiro ${ }^{1,2}$, Luísa Pinto ${ }^{1,2}$ and Nuno Sousa ${ }^{1,2^{*}}$
}

\begin{abstract}
Adult neurogenesis represents a dynamic level of modulation upon the neuroplastic properties of the mature nervous system, that is essential to the homeostatic brain function. The adult neurogenic process comprises several sequential steps, all of which subjected to an assortment of cell-intrinsic and neurogenic-niche complex regulatory mechanisms. Among these, epigenetic regulation is now emerging as a crucial regulator of several neurogenesis steps. In particular, the active regulation of hippocampal neurogenesis and its repercussions in global hippocampal function are of special interest for the biomedical field, since imbalances at this level have been strongly related to the precipitation of several neuropsychyatric disorders, such as depression. Indeed, growing evidence supports that the detrimental effects on adult hippocampal neurogenesis, that have been associated with depression, might be epigenetically-mediated. Therefore, understanding the epigenetic regulation of the neurogenic process may provide a link between neurogenesis imbalances and the deterioration of the behavioural and cognitive domains frequently affected in depression, thus contributing to unravel the complex pathophysiology of this disorder. Here, we outline some of the major epigenetic mechanisms contributing to the regulation of hippocampal neurogenesis and discuss several lines of evidence supporting their involvement on the development of imbalances in the neurogenic process, often correlated to behavioural and cognitive deficits commonly observed in major depressive disorder.
\end{abstract}

Keywords: adult neurogenesis, depression, epigenetics, antidepressants, hippocampus, dentate gyrus

\section{Adult neurogenesis: the neurogenic process and its epigenetic regulation Neurogenesis in the adult brain}

The beauty of research is that it ultimately defeats all established dogmas, even though some take very long to fall. Cajal's decree concerning the immutability of the central nervous system (CNS) has been reviewed and updated during the last decades, due to mounting evidence that substantiates the regenerative potential and plasticity of the CNS. Despite the initial reluctance manifested towards the first reports of post-natal neurogenesis, it is now well established that neurogenesis, a process that comprises the generation, differentiation and integration of new neurons in the preexisting brain neuronal networks, occurs in the adult brain, prevailing throughout life in specific brain areas, where neurons are persistently generated [1,2]. Such

\footnotetext{
* Correspondence: njcsousa@ecsaude.uminho.pt

'Life and Health Sciences Research Institute, School of Health Sciences,

University of Minho, Campus de Gualtar 4710-057 Braga, Portugal

Full list of author information is available at the end of the article
}

spatially defined brain regions where neurogenesis occurs display a permissive microenvironment for the maintenance and differentiation of neural stem cells and to their proliferation. Currently, two neurogenic brain regions are broadly recognized in the mammalian adult brain: the subgranular zone (SGZ) of the hippocampal dentate gyrus (DG) and the subependymal zone (SEZ) in the lateral ventricles.

In the hippocampal formation, the precursor cell population resides throughout the SGZ, with specific gradients [3]. After being generated in the SGZ, newly-born cells become committed to a neuronal lineage and migrate into the granule cell layer (GCL), where they mature to become excitatory glutamatergic granule neurons $[4,5]$. In the SEZ the precursor cells are mostly found in the anterior segment of the walls of the lateral ventricles. Here, newlyborn precursor cells generate neuroblasts that will migrate along the rostral migratory stream (RMS), reaching the olfactory bulb $(\mathrm{OB})$, where they fully differentiate mostly into granule inhibitory interneurons [6,7]. In addition to

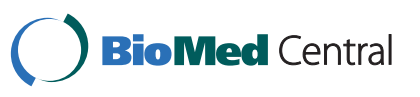


these two consensually accepted neurogenic regions, some authors have presented evidence that neurogenesis occurs in other brain areas, including the striatum [8], the cortex $[9,10]$, the amygdala [11] and the hypothalamus [12,13]; however, as these results are still disputable [14,15], further studies are needed in order to elucidate if other neurogenic niches are indeed present in the adult brain.

Although it is now indisputably accepted that neurogenesis occurs in the adult brain, its functional relevance remains to be fully established. While it is clear that this phenomenon is confined to very discrete brain regions, the generation of new neurons in the post-natal period constitutes a new dimension of plasticity, with both direct and indirect impact on neuronal remodelling and repair, that is now regarded by the biomedical field as a promising therapeutical target in several neuropathological contexts. Notably, abnormal alterations in the hippocampal neurogenesis process have been implicated in an assortment of neuropsychiatric disorders [16-18]. Several research works, seeking to unveil the biological mechanisms underlying these disorders, became comprehensive studies about the hippocampal neurogenic process. Quite surprisingly, the functional relevance of adult neurogenesis in the SEZ has not yet been directly related to any specific neuropathological condition.

\section{A brief overview on adult hippocampal neurogenesis}

Integration of newly-born hippocampal neurons into preestablished neural networks seems to be achieved through highly regulated sequential steps: proliferation of neural stem cells, generation of amplifying progenitors, cell migration and, finally, maturation in the definitive destination with axon and dendrites formation and establishment of new synapses with preexisting surrounding cells [19-21] (Figure 1). This process of post-natal neurogenesis largely recapitulates the embryonic one, with the major difference that new neurons have to undergo these steps in an already mature microenvironment, having to integrate preexisting neural circuits.

The adult hippocampus SGZ contains an heterogeneous precursor cell population, distinctly identifiable through a particular set of molecules that each cell type expresses. The quiescent neural progenitors (QNPs) are believed to be the multipotent stem cells residing on the hippocampus $[5,22]$; they are also known as neural stem cells (NSCs) or, according to an alternative nomenclature, type-1 progenitor cells. Having both morphological and antigenic glial properties $[23,24]$, they can be further distinguishable into two classes according to their spatial orientation: horizontal astrocytes (hA) and radial astrocytes (rA). These cells divide asymmetrically giving rise to daughter cells known as transiently amplifying neural progenitors (ANPs; also generally designated as type- 2 progenitor cells). This phase of the neurogenic process comprises the emergence of the first indications of neuronal or non-neuronal lineage commitment [21], being for such reason, a decisive checkpoint in the determination of neural progenitors cell-fate. Anomalous alterations in this phase of the neurogenic process often result in long-term neuropathological traits [25]. Studies have showed that ANPs are highly mitotic $[1,25]$, dividing symmetrically and giving rise to neuroblasts (NBs; also named type-3 progenitor cells). Neuroblasts are intermediate precursors in the generation of new granule neurons, expressing the microtubule associated protein doublecortin (DCX) that will be crucial for further maturation and migration $[19,26]$. Once the newborn cell becomes a neuroblast, it exits the proliferation cycle, and migrates towards its final destination in the GCL. Here, the newly-born cells will fully maturate, elongating their axons and establishing new functional connections, eventually becoming a mature granule neuron. The time window that takes to a newborn cell to be fully mature and integrated in the preexisting neural network is typically referred to be approximately five weeks $[27,28]$; however, some authors claim that the entire period of adult neurogenesis can take as much as 7 weeks [29,30], as this is the time required by the new neurons to become electrophysiologically indistinguishable from the remaining neuronal population.

Importantly, neurogenesis is a fine tuned process, rather than a mass phenomenon, during which most newborn cells are eliminated [31,32]. The mechanisms that regulate this neurogenic process are still to be fully understood, but recently, several studies proposed a complex epigenetic orchestration of adult hippocampal neurogenesis.

\section{Epigenetic orchestration of adult neurogenesis}

Functional and structural chromatin properties are actively regulated in hippocampal NSCs. In fact, and despite being a relatively recent concept in the neuroscience field, the importance of epigenetics on the fine regulation of proliferation, fate specification and differentiation of NSCs is now becoming to be recognised as fundamental for the balanced production of new neuronal and glial cells, necessary for the homeostatic brain function. Therefore, it becomes gradually evident that both extracellular signalling and intracellular epigenetically regulated gene expression programs are dynamically involved in adult neurogenesis. Notably, the intracellular epigenetic program regulating adult neurogenesis is proposed to be quite similar to the epigenetic regulation occurring during development, but is also determined by a myriad of new extrinsic physiological and environmental stimuli [33], that allow the alignment of neurogenesis with the external requests. Even though there is still much to be known, a global picture regarding the epigenetic orchestration of adult neurogenesis commences to emerge (Figure 2). 


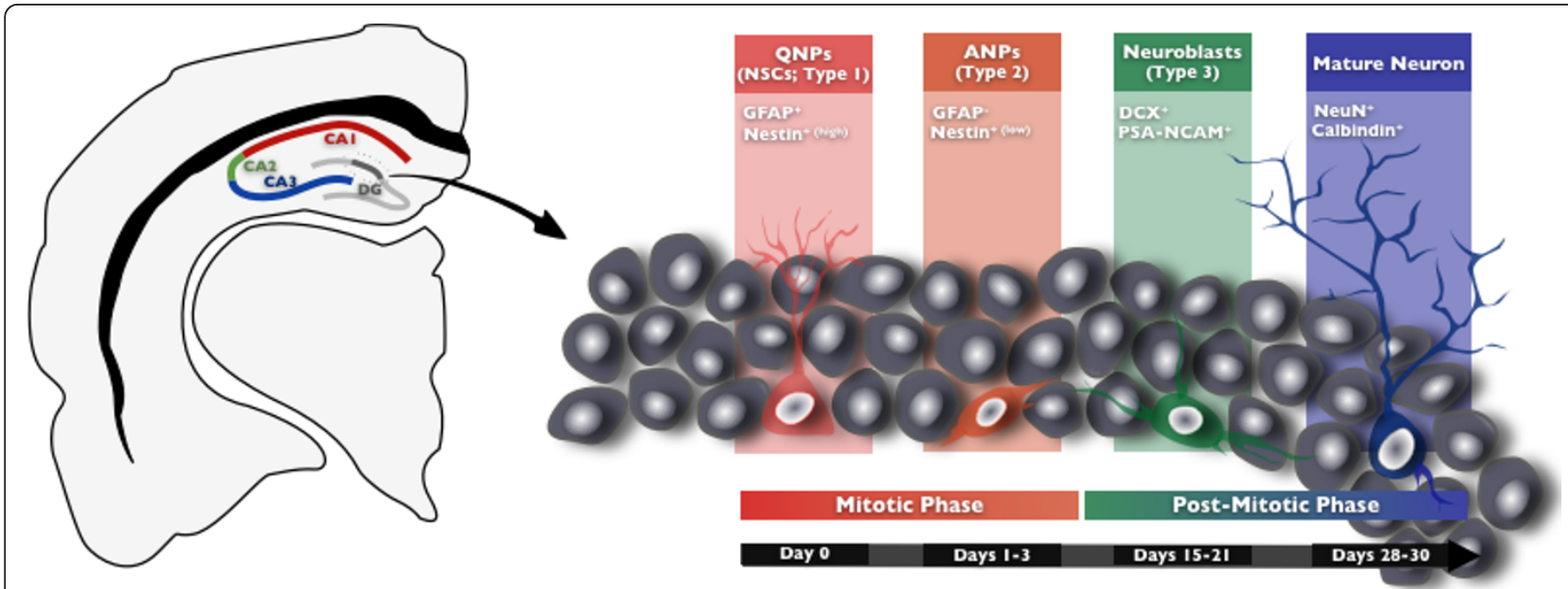

Figure 1 Neurogenesis in the dentate gyrus (DG) of the adult rodent hippocampus. The adult neurogenic process encompasses several highly regulated sequential steps. The process begins with the asymmetrical division of neural stem cells (NSCs), also named quiescent neural progenitors (QNPs or type 1 progenitors), giving rise to amplifying neural progenitors (ANPs or type 2 progenitors). ANPs start to exhibit the first signs of cell-lineage commitment and eventually exit the mitotic phase to become neuroblasts (type 3 progenitors). The neuroblasts will then differentiate and migrate towards its final destination where they will fully maturate into granular neurons and establish synapses within preexisting circuits. Each cell stage can be distinctively identifiable by cell markers, some of which are indicated. It is currently assumed that the entire process of adult neurogenesis takes around 4 to 5 weeks. (GFAP - Glial fibrillary acidic protein; DCX - Doublecortin; PSA-NCAM Polysialylated-neural cell adhesion molecule: NeuN - Neuronal Nuclei)

Epigenetic regulation is implicated in the first stages of adult neurogenesis, by promoting the maintenance of the self-renewal potential of adult NSCs. Molofsky and colleagues [34] highlighted the importance of the trithorax (trxG) and the functionally antagonistic polycomb (PcG) groups of proteins on such regulatory function. The trxG and PcG proteins are chromatin modifier complexes that will, respectively, activate or silence the targeted loci, maintaining such transcriptional state for several cell divisions [35-37]. In particular, the PcG zinc-finger protein Bmi1 has been identified as an important epigenetic regulator, promoting $\mathrm{H} 3 \mathrm{~K} 27$ methylation and repressing the expression of the cyclin-dependent kinase inhibitor gene p16 (Ink4a); as a consequence the proliferative rate of adult NSCs is maintained [38,39]. In addition, MethylCpG binding protein 1 (Mbd1) also appears to be involved in adult NSCs self-renewal in the SGZ. So far, this is the only Mbd protein reported to be specifically involved in hippocampal neurogenesis; Mbd1 deficient mice, although without noticeable developmental deficits, have reduced NSCs proliferation that correlate with cognitive impairments in spatial memory [40]. This protein binds to the promoter region of the gene encoding the mitogen Fibroblast Growth Factor 2 (Fgf2), controlling its expression on adult NSCs of the SGZ. Regulation of the expression of Fgf2 provides the precise control of the timing for the cell to exit the proliferation cycle and to initiate its differentiation [41]. In addition, the MYST family histone acetyltransferase Querkopf (Qkf or Myst4), whose expression was recently described in the adult hippocampus [42], also appears to be involved in the regulation of adult NSCs self-renewal, as NSCs isolated from Qkf mutant mice exhibited a reduced selfrenewal capacity [43]. It is important to mention that these epigenetic regulators involved in the adult NSCs maintenance, operate together with several other epigenetic protein regulators such as DNA methyltransferases (DNMTs), histone acetyltransferases (HATs), histone deacetylases (HDACs), and histone methyltransferases (HMTs), that will actively participate in further regulatory steps of the neurogenic process.

When exiting the mitotic phase, neural progenitor cells will eventually become committed to a specific neural cell lineage and start to differentiatiate. Neuronal or glial lineage commitment of NSCs involves a temporal-defined mutual regulation of several gene batteries. Commitment to a neuronal cell-fate, for instance, involves the repression of gliogenic genes; the alternative scenario of glial differentiation, requires the inhibition of genes responsible for neuronal specification. This is achieved through transcriptional and epigenetic regulation, that will integrate also the cell response to the extrinsic environment. In this context, HDACs and HATs are believed to exert an important role in the transduction of physiological signals to the stem cell genome, activating or repressing specific gene programs in NSCs. In general, HDACs catalyze the deacetylation of nucleossomes, that become highly condensed, obstructing the access of transcriptional activation factors to their binding sites and, therefore, resulting in transcriptional repression. In contrast, HATs catalyze the opposite 


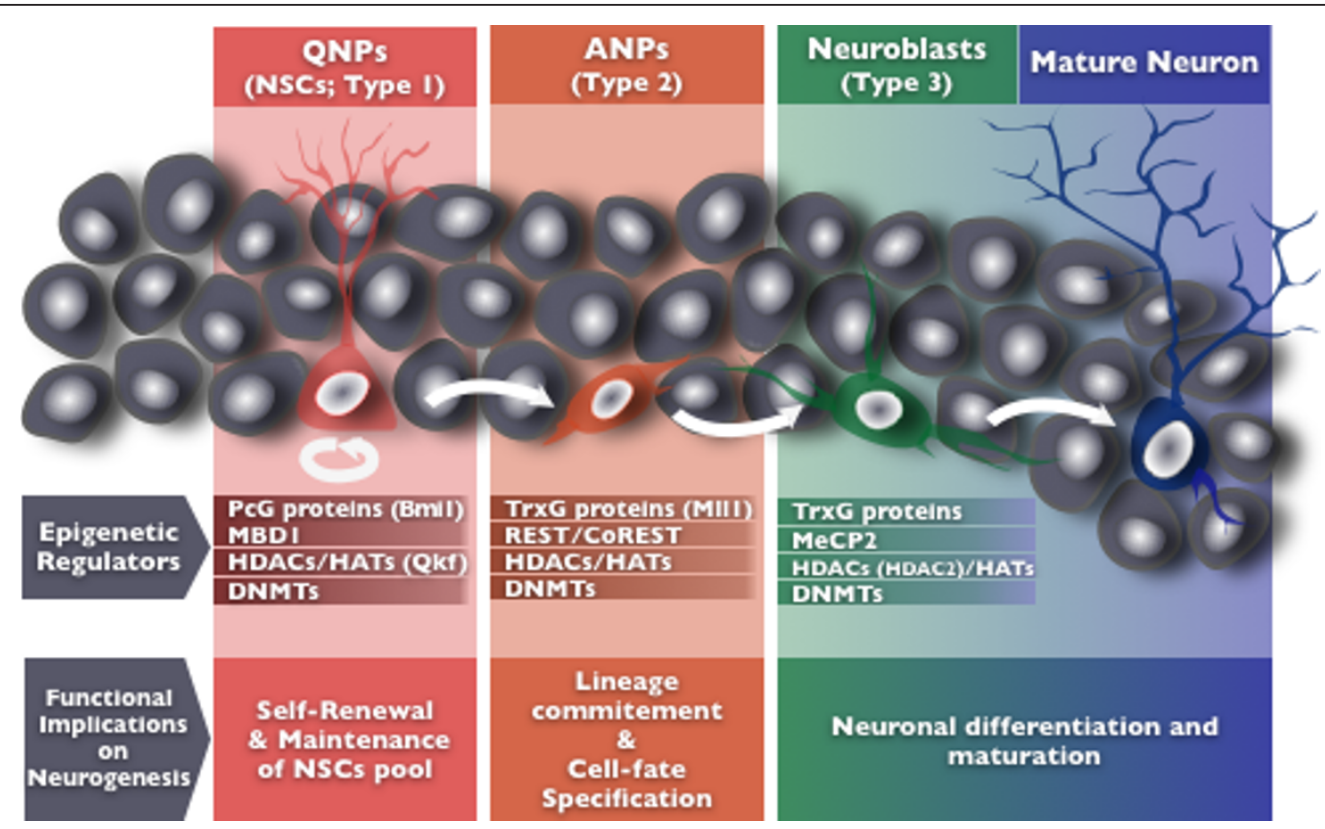

Figure 2 Epigenetic regulators of the adult hippocampal neurogenic process. The adult hippocampal neurogenic process is subjected to a complex epigenetic regulation, with important functional implications. Different types of epigenetic regulators have been identified, including PCG and TrxG protein complexes, MBDI, the REST/CoREST complex, MeCP2, HDACs, HATs and DNMTs, specifically involved in the fine tunning of the proliferation and specification of neural progenitors, as in the differentiation and maturation of the newborn neurons. Epigenetic regulators, such as the PCG protein Bmi1 and the methyl-binding protein MBD1 are involved in the regulation of the initial steps of neurogenesis, participating in NSCS self-renewal and maintenance. Later on the neurogenic process, the transcriptional activation of specific gene batteries by TrxG proteins like MII1, together with the action of chromatin remodeling complexes such as the REST/CoREST complex and its molecular partners will allow the progenitor cells to exit the proliferation cycle and become committed to a neural cell lineage. Finally, the action of regulators such as $\mathrm{MeCP} 2$, will contribute to post-mitotic neuronal differentiation and maturation. Some epigenetic regulators, such as HDACs, HATs and DNMTs are involved in several regulatory checkpoints of the adult neurogenic process, integrating several protein regulatory complexes involved in the transcriptional activation of pro-neurogenic genes.

reaction, resulting in global nucleossomal relaxation and, consequently, in increased transcriptional activity [44]. A hint for the importance of these epigenetic modifiers on the neurogenic process, was provided when administrating HDACs inhibitors (HDACis), such as trichostatin A or valproic acid (VPA) on rats. Using such experimental approach, it was shown that HDACis promote neuronal differentiation of adult neural progenitor cells [45]. In addition, at this differentiation stage other proteins, such as trxG proteins, exert their modulatory actions which will ultimately determine cell-fate specification. One interesting example is provided by the trxG family member, Mll1 (mixed-lineage leukaemia 1), which is a H3K4 HMT. Mll1-deficient NSCs retain the capacity to proliferate and fully differentiate into glial lineages, but neuronal differentiation becomes severely compromised. Therefore, Mll1 seems to be required to mediate the transition from a silenced to an active transcriptional state in key loci of postnatal neural precursors necessary for the induction of neurogenesis [46].

Although several other epigenetic regulatory players in cell-fate specification have been identified, it becomes difficult to consider a unifying model through which all epigenetic regulatory actions are coordinated. Nevertheless, a promising candidate for orchestrating these epigenetic events is the DNA binding protein REST (Repressor Element 1 Silencing Transcriprition Factor). REST was first described in $199 \overline{5}$, as a repressor of neuronal genes containing a 23 bp conserved sequence, known as RE1 (Repressor Element 1, also named neuron-restrictive silencing factor, or NRSF) $[47,48]$. This transcription factor coordinates the action of several epigenetic complexes that are required when switching from the undifferentiated stem cell state through the stages of neuronal or glial cellfate specification [49-51]. After binding to DNA, REST orderly recruits several DNMTs, HMTs, HATs, HDACs, MBDs, co-regulators (CoREST) and cell-cycle proteins, promoting shifts in the overall transcriptional state of specific gene batteries in a cellular context-sensitive manner $[49,52]$. The recruited epigenetic modulatory proteins, together with specific non-coding RNAs, interact with REST, allowing the precise control of the cellular events that lead to neural progenitors subtype specification [53,54].

Epigenetic regulation is now known to be also implicated in the final maturation of newborn neurons; at this 
stage it has an important role in promoting the integration of newly-born neurons into the preexisting neural networks. HDACs and HATs appear to be once again decisive for neuronal maturation and early synaptogenesis $[55,56]$. Using mice deficient on HDAC1 or HDAC2, it was shown that a decreased activity of both enzymes in immature hippocampal neurons triggers excitatory synapse maturation; however, exclusive inhibition of HDAC2 triggers the opposite effect and promotes reduced excitatory synaptic activity $[55,57]$. Another important epigenetic regulator in the $S G Z$ granule neurons is the methyl-CpG-binding protein 2 (MeCP2). Interestingly, in postmitotic SGZ neurons, the expression of brain-derived neutrophic factor (BDNF), a neurotrophin actively involved in dendritic growth and spine maturation, is associated to reduced DNA methylation and to the release of a chromatin repressive complex comprising $\mathrm{MeCP} 2$, in which these cells are highly enriched [58,59]. Furthermore, it has been demonstrated, using knockout mice, that MeCP2 deficiency causes severe deficits in the maturation of newborn neurons in the SGZ, including delayed differentiation and reduced dendritic spine density [60]. In addition, BDNF expression (as well as FGF) can be controlled by other epigenetic regulators such as the DNA-damage inducible protein $45 \mathrm{~b}$ (Gadd45). This protein is an activity-induced immediate early gene, and its transcription is sensitive to various stimuli that increase neurogenesis by DNA demethylation in mature neurons of neurogenic niches, contributing to the paracrine secretion of neurotrophic factors (BDNF and FGF) that control key processes in adult neurogenesis, including neuronal and dendritic maturation $[61,62]$.

Finally, it is important to note that this epigenetic regulation is not only mediated through cell-intrinsic mechanisms, and that epigenetic mediators are likely stable transducers of extracellular signals (e.g. neuronal activitydependent or from adjacent glial or endothelial cells) into the regulation of all phases of neurogenesis. The paracrine action of MeCP2 and Gadd45b are amongst the best known examples of this integration of intrinsic and extrinsic signals relevant for neurogenesis regulation [63-65]. Noticeable, these epigenetic regulators exert a complex orchestration of neurogenesis and, therefore, it is plausible that a deregulation of this epigenetic regulatory process is implicated in the neurogenic impairments observed in several neuropsychiatric disorders.

\section{Role of epigenetic (de)regulation in the ethiopathogenesis of depression: impacts on neurogenesis}

Adult hippocampal neurogenesis on the pathophysiology of depression

Adult hippocampal neurogenesis represents an important, and formerly underestimated, form of neuroplasticity, namely in the hippocampal formation, a brain structure involved in several neuropsychyatric disorders $[17,18,66]$. Indeed, there is now mounting evidence for the implication of adult hippocampal neurogenesis in the pathophysiology of several neuropsychiatric disorders, a topic extensively reviewed elsewhere $[16,19,67]$. Perhaps one of the most striking findings in this scientific context was the involvement of adult neurogenesis imbalances in the pathophysiology of major depressive disorder (MDD), as in the action of several antidepressant drugs, thus leading to the substantiation of the so called "neurogenic hypothesis of depression" $[68,69]$. In fact, several studies have linked reduced neurogenesis to depressive-behaviour and even to the action of several antidepressant drugs [70-73]. Indeed, during the last decade it became obvious the scientific insufficiency of the previously predominant neurochemical-based hypothesis to explain the precipitation of depression, with several authors putting forward alternative underlying mechanisms for the ethiopathogenesis of this disorder [74-76].

Impaired neuronal plasticity is increasingly viewed as central in the ethiopathogenesis of depression. In fact, during the last two decades a significant number of studies in this field revealed cell loss and neuronal atrophy, particularly in brain loci relevant for emotional behaviour control. Several mechanisms were proposed to be responsible for this neuronal atrophy, namely glucocorticoid and glutamate toxicity for both glia and neurons [77], decreased neurotrophic factors expression $[78,79]$, and, more interestingly, decreased neuroplasticity, including dendritic atrophy in the hippocampus in some executive-function brain centres as the prefrontal cortex (PFC) (Bessa et al., 2009b) in animal models for depression. However the most robust link between impaired neuroplasticity and MDD derives from a large number of studies reporting impaired neurogenesis in subjects displaying depressivelike symprtoms [80-82]. Further support to the association of hippocampal neurogenic control and depression, derives from the analysis of the effects of some antidepressants (ADs) in the adult neurogenic process. Counteracting the adverse effects of some of the inducing factors of MDD, ADs bolster neurogenesis in the mammalian hippocampal DG. This pharmacological enhancement of neurogenesis was reported with different classes of ADs, including selective serotonin reuptake inhibitors (SSRIs), monoamine oxidase inhibitors (MAOIs), tricyclic agents and even with putative ADs [70-73,79,83]. Consistent with the results obtained in animal models of depression, ADs also exert this pro-neurogenic effect in non-human primates and humans [84,85].

Lastly, a third link between MDD and hippocampal neurogenesis, is reflected in the functional importance of the adult neurogenic process in some of the behavioural domains commonly affected in depressive patients, such 
as mood, anxiety and cognition [86-89]. However, the initial proposals claiming that the neurogenic modulatory effects of ADs were responsible for all the behavioural improvements observed after chronic treatment with these drugs is an oversimplification as demonstrated by several studies [70,71,90-93]. In fact, we have demonstrated that the short-term mood-improving actions of antidepressants depended on neuronal remodelling, rather than on neurogenesis [70]. This is not surprising when considering that the pro-neurogenic effects mediated by ADs would not be of neurobiological significance at short-term, since a newborn neuron takes approximately a 5 weeks period to be fully differentiated and integrated in the neuronal circuitry of the adult DG. However, as the majority of studies focus on short-term analysis, one cannot rule out the possibility that the functional contribution of AD-induced neurogenesis will only take place at the long-term. In fact, preliminary data from our lab suggests that despite triggering an immediate pro-neurogenic response, the neurobiological importance of this effect of ADs becomes only significant later on the course of the disease, since the artificial suppression of neurogenesis by the anti-mitotic agent methylazoxymethanol (MAM) significantly compromises behavioural and cognitive long-term recovery, an effect that can be counteracted by ADs treatment (unpublished data).

\section{Epigenetic (de)regulation of adult neurogenesis as a possible precipitator of depression}

During the last decade, compelling evidence has emerged for the participation of epigenetic regulatory mechanisms in adult hippocampal neurogenesis. Therefore, it appears reasonable to conceive the hypothesis that dysfunction in epigenetic regulatory mechanisms might mediate the neurogenic imbalances responsible for some neuropsychiatric conditions, such as depression. Although this idea is still controversial, several lines of research suggest that different epigenetic regulatory mechanisms of adult neurogenesis are affected in animal models of depression (Figure 3).

A paradigmatic case supporting this hypothesis is the already mentioned methyl-CpG-binding protein MBD1. In fact, an elucidative work conducted by Allan and colleagues [94], showed that Mbd1-deficient mice, besides having decreased NSCs proliferation, as already described by Zhao et al., also exhibit significant deficits in several behavioural dimensions relevant for depression: increased anxious phenotype, detected in both elevated plus maze and light-dark preference tests; behavioural despair, observed in the forced swimming test; and cognitive deficits manifested during the execution of Morris water maze spatial learning tasks [94].
Another example derives from studies focused on MRG15, an active component of HDACs complexes, such as HDAC2 [95,96]. Indeed, Mrg15-deficient mice present significant deficits in proliferation of neural progenitors and in their subsequent differentiation [97]. Interestingly, HDAC2 has been identified as a negative regulator of memory, as HDAC2-overexpressing mice presented decreased spine density and synaptic plasticity, that correlates with reduced memory formation [56]. These results confirm the involvement of this histone post-translational modifier protein in controlling both the adult neurogenic process and some of the associated cognitive abilities, also typically affected in stress-related disorders, such as MDD.

Moreover, epigenetic regulators directly involved in post-mitotic neuronal maturation and differentiation, have also been associated with several behavioural and cognitive impairments present in several neuropsychiatric disorders. Work from Adachi et al. [98], for instance, has demonstrated that MeCP2 may interfere in neurological pathways that mediate heightened anxiety. DNMTS, active epigenetic regulators of adult neurogenesis, participating throughout all its phases, are also dynamically involved in blocking memory formation $[99,100]$. Curiously, DNMT3b, an enzyme responsible for de novo DNA methylation, has been reported to have an increased expression in depressive suicide completers. Interestingly, this increase was significantly more pronounced in women, a result that is in accordance with the gender preference of MDD (twice more prevalent in women) [101].

In addition, pharmacological and non-pharmacological treatments of depression, such as ADs and electroconvulsive shock (ECS) therapy, respectively, provide additional endorsement of the hypothesis that the neurogenic precipitation of depression might be, at least partially, epigenetically mediated. In fact, imipramine, a tricyclic agent with a well described pro-neurogenic action $[70,79]$, has behavioural improving actions in a socially defeated mice model, that correlates with downregulation of HDAC5 in the hippocampal region [102]. In contrast, viral-mediated overexpression of HDAC5 counteracts the effects of chronic imipramine treatment in reversing depressive-like behaviour. Interestingly, HDAC5 participates in adult neurogenesis regulation, controlling both maturation and survival of newborn neurons [103]. Interestingly, VPA clinical effectiveness as a mood stabiliser has been correlated to its neurogenic enhancement effect $[104,105]$. Additional studies showed that VPA, in conjugation with sodium butyrate, when administered alone or in combination with the antidepressant fluoxetine, improves performance in animal models of behavioural despair [106,107]. Considering that VPA is also an HDACi, and that HDAC inhibition is known to drive adult hippocampal neurogenesis [45], these studies highlight the importance of the pharmacological 


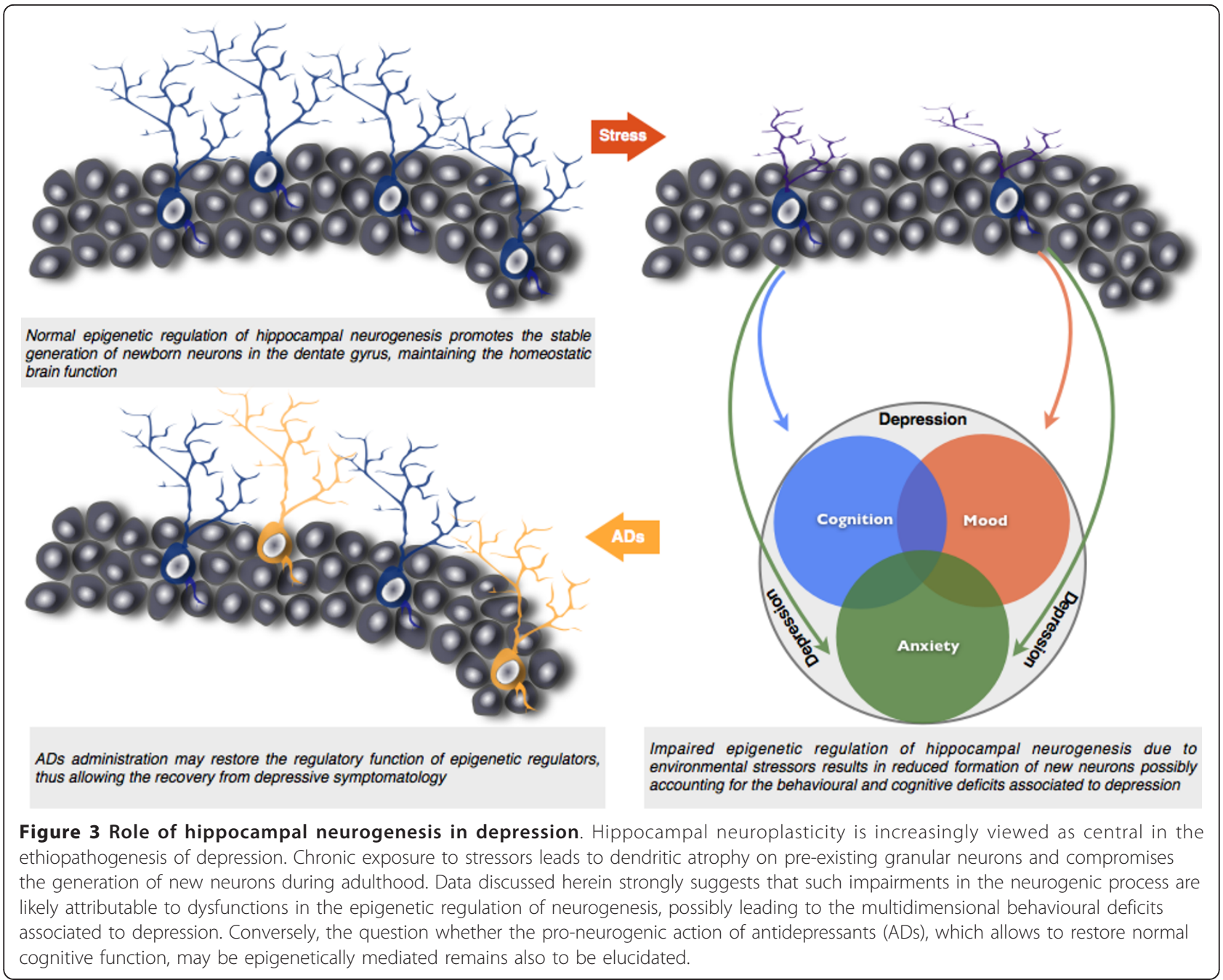

modulation of epigenetic regulators involved in adult neurogenesis to the efficiency of some ADs to ameliorate depressive behaviour. Non-pharmacological treatment of MDD, through ECS therapy, is also known to enhance hippocampal neurogenesis [108]. Interestingly, Gadd45b-deficient mice fail to reveal the ECS-induced increase in adult neurogenesis [62], suggesting that the pro-neurogenic action of ECS therapy, important for reversing depressivelike behaviour, might be epigenetically-mediated by Gadd45b.

Finally, it is worth to mention that this epigenetic regulation is also implicated in the vulnerability to stress, enhancing the susceptibility to stress-related disorders, in which depression is included. The role of such epigenetic mechanisms has recently been highlighted. Indeed, REST4, a splicing variant of the epigenetic orchestrator REST, has been shown to have an increased expression in rats exposed to stress early in life; importantly, these animals display enhanced susceptibility to stress and increased susceptibility to depressive-like behaviour
[109]. In addition, KAP1, a crucial component of a repressive chromatin complex, seems also to be involved in stress vulnerability. In fact, mice with deletion of KAP1 in the forebrain exhibit high levels of anxiety-like behaviour and significant stress-induced impairments in some cognitive domains, such as attention and spatial reference memory $[110,111]$. Therefore, and although not directly involved on neurogenesis regulation, epigenetic-mediated increases in the vulnerability to precipitation factors of depression, such as stress, may lead to an accentuation of the detrimental effects upon the neurogenic process dynamics and its regulatory mechanisms, thus favouring the development of deficits at the behavioural and cognitive levels.

\section{Conclusions - Towards a neuro-epigenetic hypothesis of depression?}

The dynamic and environmentally driven modulation of neuroplasticity in the adult brain plays a crucial role in the ethiopathogenesis of depression. As discussed 
herein, the last two decades provided several lines of evidence supporting the implication of hippocampal neurogenesis in the maintenance of the homeostatic brain function and its importance in the pathophysiology of MDD (Figure 4). However, approaches to this topic have been largely descriptive and the field still lacks an integrative perspective regarding genes and molecular determinants influencing neurogenesis in the installation of such neuropathological conditions; importantly the same holds true for the positive action of ADs in this process.

Our comprehension of how adult neurogenesis is regulated begins now to be complemented with new insights into the complex epigenetic orchestration of this phenomenon, a regulatory dimension that is relatively new in neuroscience research. Yet, the scattered evidence gathered so far, opens a new dimension for unravelling the mechanistic explanation for the interplay of genes and environment

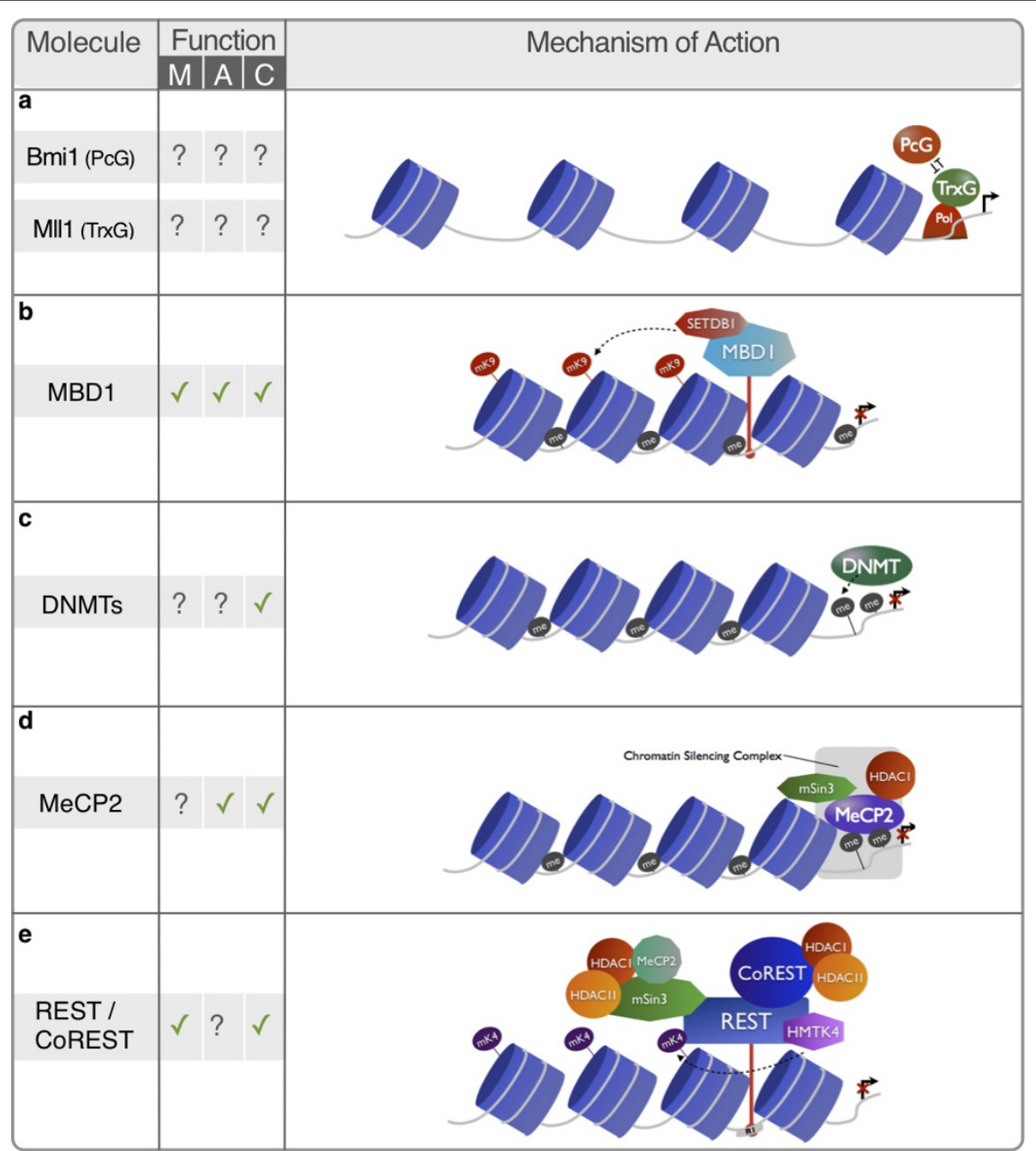

Figure 4 Epigenetic regulators of neurogenesis on the development of pathological behavioural traits. Several epigenetic regulators have been implicated in the control of the adult neurogenic process. As such, it is likely that the mechanistic action of these molecules is implicated in the behavioural dimensions commonly affected in depression: Mood (M), Anxiety (A) and Cognition (C). " $\checkmark$ " indicates that such involvement has been described; "?" indicates that the implications of the molecule are unknown or still unclear. a PcG and TrxG protein complexes silence or activate, respectively, the transcription of target genes and have been implicated in the control of the neurogenic process; however, repercussions at the behavioural level still remain to be described; $\mathbf{b}$ MBD1 action is associated with the histone-lysine N-methyltransferase SETDB1, silencing target genes; notably, deficits in this molecule has been associated to deficits in all three behavioural dimension of depression. $\mathbf{c}$ DNMTs participate in the regulation of a broad range of neurogenesis processes, being its action strongly related to deficits in learning and memory; although some studies suggest that they might be also involved in the transcriptional regulation of pathways associated to mood and anxiety, such correlation needs to be further endorsed; d MeCP2 integrates a major chromatin silencing complex comprising several others epigenetic regulators, such as HDAC1, involved in the trancriptional regulation of several genes. Deficits in this molecule have been correlated with cognitive and anxiety deficits, although no deficits in mood have been consistently described; e The REST/COREST chromatin remodeling complex has been proposed as a major orchestrator of the action of several epigenetic regulators, such as HDAC 1 and 2, MeCP2 and the histone methyltransferase K4. Impairments in mood an cognition have been associated with REST and its molecular partners, although no implication have been described relating this molecule to anxiety. 


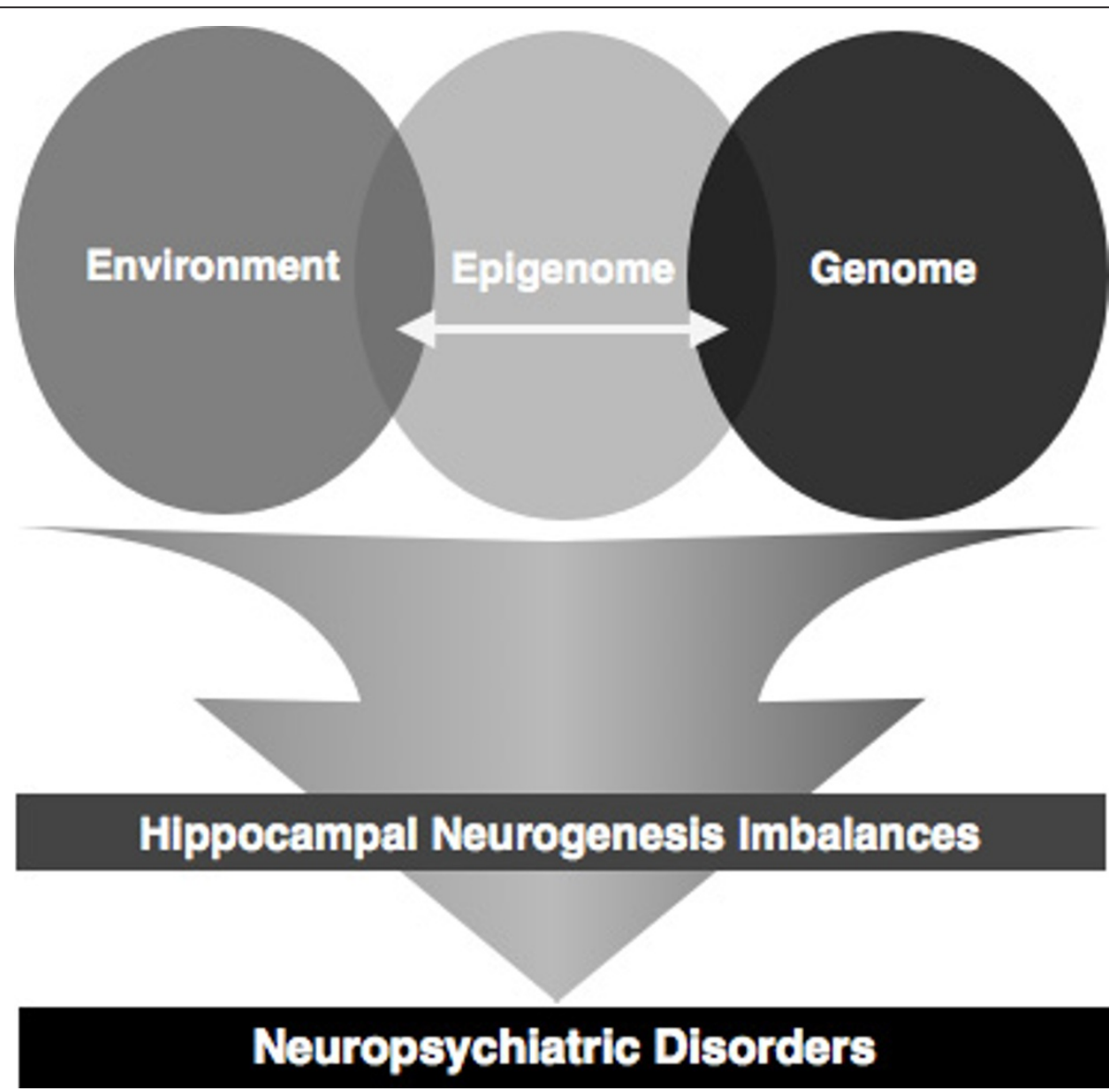

Figure 5 A neuro-epigenetic hypothesis for the ethiology of neuropsychiatric disease. Such hypothesis states that Impairments in the environment-genome interplay mediated by epigenetic regulation may account for the imbalances observed at the hippocampal neurogenesis level, thus triggering the onset of the symptomatology typical of such disorders.

that is central in several neuropsychiatric pathological scenarios. In fact, studies in this area endorse the role of epigenetic mechanisms as transducers of the environmental signals into transcriptional outcomes, strongly suggesting that they may participate in the precipitation of experience-dependent psychiatric disorders, like depression, and simultaneously mediate the pro-neurogenic action of commonly prescribed ADs. Therefore, epigenetic mechanisms begin to emerge as mediators through which environment modulates neurogenesis with long-lasting and stable repercussions in several behavioural and cognitive domains (Figure 5). In this perspective, it becomes plausible that by reverting the pathological effects on epigenetic key regulators, one can counteract the deleterious effects of stress and other precipitators of depression, thus restoring normal neurogenic function. Hence, future research focused on dissecting the epigenetic pathways that modulate the adult neurogenesis process will be decisive to further unravel the neurobiological basis of depression and may pave the way to the development of novel therapies and to the discovery of new therapeutical targets in this pathological context.

\section{Author details}

${ }^{1}$ Life and Health Sciences Research Institute, School of Health Sciences, University of Minho, Campus de Gualtar 4710-057 Braga, Portugal. ${ }^{2}$ ICVS/3B's - PT Government Associate Laboratory, Braga/Guimarães, Portugal.

\section{Authors' contributions}

AP drafted the manuscript. LP and NS revised the manuscript and coordinated the work. All authors read and approved the final manuscript.

\section{Conflicts of interests}

The authors declare that they have no competing interests.

Received: 8 April 2011 Accepted: 1 November 2011

Published: 1 November 2011

\section{References}

1. Doetsch F, Caillé I, Lim Da, García-Verdugo JM, Alvarez-Buylla a: Subventricular zone astrocytes are neural stem cells in the adult mammalian brain. Cell 1999, 97:703-716.

2. Gage FH: Neurogenesis in the adult brain. The Journal of neuroscience: the official journal of the Society for Neuroscience 2002, 22:612-613. 
3. Silva R, Lu J, Wu Y, Martins L, Almeida OF, Sousa N: Mapping cellular gains and losses in the postnatal dentate gyrus: implications for psychiatric disorders. Exp Neurol 2006, 200(2):321-331.

4. Brill MS, Ninkovic J, Winpenny E, Hodge RD, Ozen I, Yang R, Lepier A, Gascón S, Erdelyi F, Szabo G, Parras C, Guillemot F, Frotscher M, Berninger B, Hevner RF, Raineteau O, Götz M: Adult generation of glutamatergic olfactory bulb interneurons. Nature neuroscience 2009, 12:1524-1533.

5. Seri B, Garcia-Verdugo JM, Collado-Morente L, McEwen BS, Alvarez-Buylla A: Cell types, lineage, and architecture of the germinal zone in the adult dentate gyrus. The Journal of comparative neurology 2004, 478:359-378.

6. Chumley MJ, Catchpole T, Silvany RE, Kernie SG, Henkemeyer M: EphB Receptors Regulate Stem/Progenitor Cell Proliferation, Migration, and Polarity during Hippocampal Neurogenesis. Journal of Neuroscience 2007, 27:13481-13490.

7. Wu W, Wong K, Chen J, Jiang Z, Dupuis S, Wu JY, Rao Y: Directional guidance of neuronal migration in the olfactory system by the protein Slit. Nature 1999, 400:331.

8. Luzzati F, De Marchis S, Fasolo A, Peretto P: Neurogenesis in the caudate nucleus of the adult rabbit. Journal of Neuroscience 2006, 26:609.

9. Kodama M, Fujioka T, Duman RS: Chronic olanzapine or fluoxetine administration increases cell proliferation in hippocampus and prefrontal cortex of adult rat. Biological psychiatry 2004, 56:570-580.

10. Ohira K, Furuta T, Hioki H, Nakamura KC, Kuramoto E, Tanaka Y, Funatsu N, Shimizu K, Oishi T, Hayashi M, et al: Ischemia-induced neurogenesis of neocortical layer 1 progenitor cells. Nature Neuroscience 2009, 13:173-180.

11. Gonçalves L, Silva R, Pinto-Ribeiro F, Pego JM, Bessa JM, Pertovaara A, Sousa N, Almeida A: Neuropathic pain is associated with depressive behaviour and induces neuroplasticity in the amygdala of the rat. Exp Neurol 2008, 213(1):48-56.

12. Fowler $C D$, Liu Y, Ouimet $C$, Wang Z: The effects of social environment on adult neurogenesis in the female prairie vole. Journal of neurobiology 2002, 51:115-128.

13. Kokoeva MV, Yin H, Flier JS: Neurogenesis in the hypothalamus of adult mice: potential role in energy balance. Science (New York, NY) 2005, 310:679-683.

14. Chmielnicki E, Benraiss A, Economides AN, Goldman Sa: Adenovirally expressed noggin and brain-derived neurotrophic factor cooperate to induce new medium spiny neurons from resident progenitor cells in the adult striatal ventricular zone. The Journal of neuroscience: the official journal of the Society for Neuroscience 2004, 24:2133-2142.

15. Ehninger D, Kempermann G: Regional Effects of Wheel Running and Environmental Enrichment on Cell Genesis and Microglia Proliferation in the Adult Murine Neocortex. Cerebral Cortex 2003, 845-851.

16. Eisch AJ, Cameron Ha, Encinas JM, Meltzer La, Ming G-L, OverstreetWadiche LS: Adult neurogenesis, mental health, and mental illness: hope or hype? The Journal of neuroscience: the official journal of the Society for Neuroscience 2008, 28:11785-11791.

17. Kobayashi $\mathrm{K}$ : Targeting the hippocampal mossy fiber synapse for the treatment of psychiatric disorders. Molecular neurobiology 2009, 39:24-36.

18. Sapolsky RM: Glucocorticoids and hippocampal atrophy in neuropsychiatric disorders. Archives of general psychiatry 2000, 57:925.

19. Balu DT, Lucki I: Adult hippocampal neurogenesis: regulation, functional implications, and contribution to disease pathology. Neuroscience and biobehavioral reviews 2009, 33:232-252.

20. Kempermann G, Jessberger S, Steiner B, Kronenberg G: Milestones of neuronal development in the adult hippocampus. Trends in neurosciences 2004, 27:447-452.

21. Steiner B, Klempin F, Wang L, Kott M, Kettenmann H, Kempermann G: Type- 2 cells as link between glial and neuronal lineage in adult hippocampal neurogenesis. Glia 2006, 54:805-814.

22. Seri B, Garcia-Verdugo JM, McEwen BS, Alvarez-Buylla A: Astrocytes give rise to new neurons in the adult mammalian hippocampus. Journal of Neuroscience 2001, 21:7153.

23. Filippov V: Subpopulation of nestin-expressing progenitor cells in the adult murine hippocampus shows electrophysiological and morphological characteristics of astrocytes. Molecular and Cellular Neuroscience 2003, 23:373-382.

24. Rakic P: Elusive radial glial cells: historical and evolutionary perspective. Glia 2003, 43:19-32.

25. Encinas JM, Vaahtokari A, Enikolopov G: Fluoxetine targets early progenitor cells in the adult brain. Proceedings of the National Academy of Sciences of the United States of America 2006, 103:8233-8238.
26. Pleasure SJ, Collins aE, Lowenstein DH: Unique expression patterns of cell fate molecules delineate sequential stages of dentate gyrus development. The Journal of neuroscience: the official journal of the Society for Neuroscience 2000, 20:6095-6105.

27. Espósito MS, Piatti VC, Laplagne Da, Morgenstern Na, Ferrari CC, Pitossi FJ, Schinder AF: Neuronal differentiation in the adult hippocampus recapitulates embryonic development. The Journal of neuroscience: the official journal of the Society for Neuroscience 2005, 25:10074-10086.

28. Zhao C, Teng EM, Summers RG, Ming G-L, Gage FH: Distinct morphological stages of dentate granule neuron maturation in the adult mouse hippocampus. The Journal of neuroscience: the official journal of the Society for Neuroscience 2006, 26:3-11.

29. Ambrogini P, Lattanzi D, Ciuffoli S, Agostini D, Bertini L, Stocchi V, Santi S, Cuppini R: Morpho-functional characterization of neuronal cells at different stages of maturation in granule cell layer of adult rat dentate gyrus. Brain Res 2004, 1017(1-2):21-31.

30. van Praag H, Schinder AF, Christie BR, Toni N, Palmer TD, Gage FH: Functional neurogenesis in the adult hippocampus. Nature 2002, 415(6875):10q-1034.

31. Biebl M, Cooper CM, Winkler J, Kuhn HG: Analysis of neurogenesis and programmed cell death reveals a self-renewing capacity in the adult rat brain. Neurosci Lett 2000, 291(1):17-20.

32. Kuhn HG, Biebl M, Wilhelm D, Li M, Friedlander RM, Winkler J: Increased generation of granule cells in adult Bcl-2-overexpressing mice: a role for cell death during continued hippocampal neurogenesis. Eur I Neurosci 2005, 22(8):1907-1915

33. Ninkovic J, Götz M: Signaling in adult neurogenesis: from stem cell niche to neuronal networks. Current opinion in neurobiology 2007, 17:338-344.

34. Molofsky AV, Pardal R, Iwashita T, Park IK, Clarke MF, Morrison SJ: Bmi-1 dependence distinguishes neural stem cell self-renewal from progenitor proliferation. Nature 2003, 425:962-967.

35. Ng RK, Gurdon JB: Epigenetic inheritance of cell differentiation status. Cell Cycle 2008, 7:1173-1177.

36. Ringrose L, Paro R: Polycomb/Trithorax response elements and epigenetic memory of cell identity. Development (Cambridge, England) 2007, 134:223-232.

37. Schuettengruber B, Chourrout D, Vervoort M, Leblanc B, Cavalli G: Genome regulation by polycomb and trithorax proteins. Cell 2007, 128:735-745.

38. Fasano Ca, Dimos JT, Ivanova NB, Lowry N, Lemischka IR, Temple S: shRNA knockdown of Bmi-1 reveals a critical role for p21-Rb pathway in NSC self-renewal during development. Cell stem cell 2007, 1:87-99.

39. Fasano Ca, Phoenix TN, Kokovay E, Lowry N, Elkabetz Y, Dimos JT, Lemischka IR, Studer L, Temple S: Bmi-1 cooperates with Foxg1 to maintain neural stem cell self-renewal in the forebrain. Genes \& development 2009, 23:561-574.

40. Zhao X, Ueba T, Christie BR, Barkho B, McConnell MJ, Nakashima K, Lein ES, Eadie BD, Willhoite AR, Muotri AR, Summers RG, Chun J, Lee K-F, Gage FH: Mice lacking methyl-CpG binding protein 1 have deficits in adult neurogenesis and hippocampal function. Proceedings of the National Academy of Sciences of the United States of America 2003, 100:6777-6782.

41. Li X, Barkho BZ, Luo Y, Smrt RD, Santistevan NJ, Liu C, Kuwabara T, Gage FH, Zhao X: Epigenetic regulation of the stem cell mitogen Fgf-2 by Mbd1 in adult neural stem/progenitor cells. The Journal of biological chemistry 2008, 283:27644-27652.

42. Maisel M, Herr A, Milosevic J, Hermann A, Habisch H-J, Schwarz S, Kirsch M, Antoniadis G, Brenner R, Hallmeyer-Elgner S, Lerche H, Schwarz J, Storch A: Transcription profiling of adult and fetal human neuroprogenitors identifies divergent paths to maintain the neuroprogenitor cell state. Stem cells (Dayton, Ohio) 2007, 25:1231-1240.

43. Merson TD, Dixon MP, Collin C, Rietze RL, Bartlett PF, Thomas T, Voss AK: The transcriptional coactivator Querkopf controls adult neurogenesis. The Journal of neuroscience: the official journal of the Society for Neuroscience 2006, 26:11359-11370.

44. Mehler MF: Epigenetic principles and mechanisms underlying nervous system functions in health and disease. Progress in neurobiology 2008, 86:305-341.

45. Hsieh J, Nakashima K, Kuwabara T, Mejia E, Gage FH: Histone deacetylase inhibition-mediated neuronal differentiation of multipotent adult neural progenitor cells. Proceedings of the National Academy of Sciences of the United States of America 2004, 101:16659-16664.

46. Lim Da, Huang Y-C, Swigut T, Mirick AL, Garcia-Verdugo JM, Wysocka J, Ernst P, Alvarez-Buylla A: Chromatin remodelling factor MIl1 is essential 
for neurogenesis from postnatal neural stem cells. Nature 2009, 458:529-533.

47. Chong Ja, Tapia-Ramírez J, Kim S, Toledo-Aral JJ, Zheng Y, Boutros MC, Altshuller YM, Frohman Ma, Kraner SD, Mandel G: REST: a mammalian silencer protein that restricts sodium channel gene expression to neurons. Cell 1995, 80:949-957.

48. Schoenherr CJ, Anderson DJ: Silencing is golden: negative regulation in the control of neuronal gene transcription. Current opinion in neurobiology 1995, 5:566-571.

49. Ooi L, Wood IC: Chromatin crosstalk in development and disease: lessons from REST. Nature reviews Genetics 2007, 8:544-554.

50. Otto SJ, McCorkle SR, Hover J, Conaco C, Han J-J, Impey S, Yochum GS, Dunn JJ, Goodman RH, Mandel G: A new binding motif for the transcriptional repressor REST uncovers large gene networks devoted to neuronal functions. The Journal of neuroscience: the official journal of the Society for Neuroscience 2007, 27:6729-6739.

51. Singh SK, Kagalwala MN, Parker-Thornburg J, Adams H, Majumder S: REST maintains self-renewal and pluripotency of embryonic stem cells. Nature 2008, 453:223-227.

52. Ballas N, Mandel $\mathrm{G}$ : The many faces of REST oversee epigenetic programming of neuronal genes. Current opinion in neurobiology 2005, 15:500-506.

53. Visvanathan J, Lee S, Lee B, Lee JW, Lee S-K: The microRNA miR-124 antagonizes the anti-neural REST/SCP1 pathway during embryonic CNS development. Genes \& development 2007, 21:744-749.

54. Wu J, Xie X: Comparative sequence analysis reveals an intricate network among REST, CREB and miRNA in mediating neuronal gene expression. Genome biology 2006, 7:R85.

55. Akhtar MW, Raingo J, Nelson ED, Montgomery RL, Olson EN, Kavalali ET, Monteggia LM: Histone deacetylases 1 and 2 form a developmental switch that controls excitatory synapse maturation and function. $J$ Neurosci 2009, 29(25):8288-8297.

56. Guan J-S, Haggarty SJ, Giacometti E, Dannenberg J-H, Joseph N, Gao J, Nieland TJF, Zhou Y, Wang X, Mazitschek R, Bradner JE, DePinho Ra, Jaenisch R, Tsai L-H: HDAC2 negatively regulates memory formation and synaptic plasticity. Nature 2009, 459:55-60.

57. Jawerka M, Colak D, Dimou L, Spiller C, Lagger S, Montgomery RL, Olson EN, Wurst W, Gottlicher M, Gotz M: The specific role of histone deacetylase 2 in adult neurogenesis. Neuron Glia Biol 2010, 6(2):93-107.

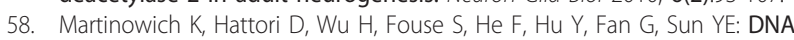
methylation-related chromatin remodeling in activity-dependent BDNF gene regulation. Science (New York, NY) 2003, 302:890-893.

59. Zhou Z, Hong EJ, Cohen S, Zhao W-N, Ho H-YH, Schmidt L, Chen WG, Lin Y, Savner E, Griffith EC, Hu L, Steen JaJ, Weitz CJ, Greenberg ME: Brainspecific phosphorylation of MeCP2 regulates activity-dependent Bdnf transcription, dendritic growth, and spine maturation. Neuron 2006, 52:255-269.

60. Smrt RD, Eaves-Egenes J, Barkho BZ, Santistevan NJ, Zhao C, Aimone JB, Gage $F H$, Zhao X: Mecp2 deficiency leads to delayed maturation and altered gene expression in hippocampal neurons. Neurobiol Dis 2007, 27(1):77-89.

61. Ma DK, Guo JU, Ming G, Song H: DNA excision repair proteins and Gadd45 as molecular players for active DNA demethylation. Cell cycle (Georgetown, Tex) 2009, 8:1526.

62. Ma DK, Jang M-h, Guo JU, Kitabatake Y, Chang M-L, Pow-Anpongkul N, Flavell RA, Lu B, Ming G-I, Song H: Neuronal activity-induced Gadd45b promotes epigenetic DNA demethylation and adult neurogenesis. Science (New York, NY) 2009, 323:1074-1077.

63. Mu Y, Lee S: Signaling in adult neurogenesis. Current Opinion in Neurobiology 2010, 20:416-423.

64. Shen $\mathrm{Q}$, Goderie SK, Jin L, Karanth N, Sun Y, Abramova N, Vincent $\mathrm{P}$, Pumiglia K, Temple S: Endothelial cells stimulate self-renewal and expand neurogenesis of neural stem cells. Science (New York, NY) 2004, 304:1338-1340.

65. Ma DK, Ming G-L, Song H: Glial influences on neural stem cell development: cellular niches for adult neurogenesis. Current opinion in neurobiology 2005, 15:514-520.

66. Hsieh J, Eisch AJ: Epigenetics, hippocampal neurogenesis, and neuropsychiatric disorders: unraveling the genome to understand the mind. Neurobiol Dis 2010, 39(1):73-84.
67. DeCarolis $\mathrm{Na}$, Eisch AJ: Hippocampal neurogenesis as a target for the treatment of mental illness: a critical evaluation. Neuropharmacology 2010, 58:884-893.

68. Duman RS: Role of neurotrophic factors in the etiology and treatment of mood disorders. Neuromolecular Med 2004, 5(1):11-25.

69. Warner-Schmidt JL, Duman RS: Hippocampal neurogenesis: opposing effects of stress and antidepressant treatment. Hippocampus 2006, 16(3):239-249.

70. Bessa JM, Palha JA, Almeida OFX, Ferreira D, Sousa N, Melo I, Marques F, Cerqueira JJ: The mood-improving actions of antidepressants do not depend on neurogenesis but are associated with neuronal remodeling. Molecular psychiatry 2009, 14:764-773, 739.

71. David DJ, Samuels BA, Rainer Q, Wang J-W, Marsteller D, Mendez I, Drew M, Craig Da, Guiard BP, Guilloux J-P, Artymyshyn RP, Gardier AM, Gerald C, Antonijevic la, Leonardo ED, Hen R: Neurogenesis-dependent and -independent effects of fluoxetine in an animal model of anxiety/ depression. Neuron 2009, 62:479-493.

72. Malberg JE, Eisch AJ, Nestler EJ, Duman RS: Chronic antidepressant treatment increases neurogenesis in adult rat hippocampus. Journal of Neuroscience 2000, 20:9104.

73. Santarelli L, Saxe M, Gross C, Surget A, Battaglia F, Dulawa S, Weisstaub N, Lee J, Duman R, Arancio O, Belzung C, Hen R: Requirement of hippocampal neurogenesis for the behavioral effects of antidepressants. Science (New York, NY) 2003, 301:805-809.

74. Castrén E: Is mood chemistry? Nature reviews Neuroscience 2005, 6:241-246

75. Pittenger C, Duman RS: Stress, depression, and neuroplasticity: a convergence of mechanisms. Neuropsychopharmacology: official publication of the American College of Neuropsychopharmacology 2008, 33:88-109.

76. Sousa N, Madeira MD, Paula-Barbosa MM: Effects of corticosterone treatment and rehabilitation on the hippocampal formation of neonatal and adult rats. An unbiased stereological study. Brain research 1998, 794:199-210

77. Abraham I, Juhasz G, Kekesi KA, Kovacs KJ: Corticosterone peak is responsible for stress-induced elevation of glutamate in the hippocampus. Stress 1998, 2(3):171-181.

78. Lee J, Duan W, Mattson MP: Evidence that brain-derived neurotrophic factor is required for basal neurogenesis and mediates, in part, the enhancement of neurogenesis by dietary restriction in the hippocampus of adult mice. Journal of neurochemistry 2002, 82:1367-1375.

79. Sairanen M, Lucas G, Ernfors P, Castrén M, Castrén E: Brain-derived neurotrophic factor and antidepressant drugs have different but coordinated effects on neuronal turnover, proliferation, and survival in the adult dentate gyrus. The Journal of neuroscience: the official journal of the Society for Neuroscience 2005, 25:1089-1094.

80. Alonso R, Griebel G, Pavone G, Stemmelin J, Le Fur G, Soubrié P: Blockade of CRF1 or V1B receptors reverses stress-induced suppression of neurogenesis in a mouse model of depression. Molecular Psychiatry 2004, 9:224-224.

81. Coe C: Prenatal stress diminishes neurogenesis in the dentate gyrus of juvenile Rhesus monkeys. Biological Psychiatry 2003, 54:1025-1034.

82. Sahay A, Hen R: Adult hippocampal neurogenesis in depression. Nature neuroscience 2007, 10:1110-1115.

83. Banasr M, Valentine GW, Li X-Y, Gourley SL, Taylor JR, Duman RS: Chronic unpredictable stress decreases cell proliferation in the cerebral cortex of the adult rat. Biological psychiatry 2007, 62:496-504.

84. Boldrini M, Underwood MD, Hen R, Rosoklija GB, Dwork AJ, Mann JJ, Arango V: Antidepressants increase neural progenitor cells in the human hippocampus. Neuropsychopharmacology: official publication of the American College of Neuropsychopharmacology 2009, 34:2376-2389.

85. Perera TD, Coplan JD, Lisanby SH, Lipira CM, Arif M, Carpio C, Spitzer G, Santarelli L, Scharf B, Hen R, Rosoklija G, Sackeim Ha, Dwork AJ: Antidepressant-induced neurogenesis in the hippocampus of adult nonhuman primates. The Journal of neuroscience: the official journal of the Society for Neuroscience 2007, 27:4894-4901.

86. Bessa JM, Mesquita AR, Oliveira M, Pêgo JM, Cerqueira JJ, Palha Ja, Almeida OFX, Sousa N: A trans-dimensional approach to the behavioral aspects of depression. Frontiers in behavioral neuroscience 2009, 3:1.

87. Clelland CD, Choi M, Romberg C, Clemenson GD, Fragniere a, Tyers $P$, Jessberger S, Saksida LM, Barker Ra, Gage FH, Bussey TJ: A functional role 
for adult hippocampal neurogenesis in spatial pattern separation. Science (New York, NY) 2009, 325:210-213.

88. Dupret D, Revest J-M, Koehl M, Ichas F, De Giorgi F, Costet P, Abrous DN, Piazza PV: Spatial relational memory requires hippocampal adult neurogenesis. PloS one 2008, 3:e1959.

89. Kee N, Teixeira CM, Wang AH, Frankland PW: Preferential incorporation of adult-generated granule cells into spatial memory networks in the dentate gyrus. Nature neuroscience 2007, 10:355-362.

90. Holick Ka, Lee DC, Hen R, Dulawa SC: Behavioral effects of chronic fluoxetine in $B A L B / C J$ mice do not require adult hippocampal neurogenesis or the serotonin $1 \mathrm{~A}$ receptor. Neuropsychopharmacology: official publication of the American College of Neuropsychopharmacology 2008, 33:406-417.

91. Jayatissa MN, Henningsen K, West MJ, Wiborg O: Decreased cell proliferation in the dentate gyrus does not associate with development of anhedonic-like symptoms in rats. Brain research 2009, 1290:133-141.

92. Sapolsky RM: Is impaired neurogenesis relevant to the affective symptoms of depression? Biological psychiatry 2004, 56:137-139.

93. Singer BH, Jutkiewicz EM, Fuller $C L$, Lichtenwalner RJ, Zhang H, Velander AJ, Li X, Gnegy ME, Burant CF, Parent JM: Conditional ablation and recovery of forebrain neurogenesis in the mouse. The Journal of comparative neurology 2009, 514:567-582.

94. Allan AM, Liang X, Luo Y, Pak C, Li X, Szulwach KE, Chen D, Jin P, Zhao X: The loss of methyl-CpG binding protein 1 leads to autism-like behavioral deficits. Access 2008, 17:2047-2057.

95. Bhaumik SR, Smith E, Shilatifard A: Covalent modifications of histones during development and disease pathogenesis. Nature structural \& molecular biology 2007, 14:1008-1016.

96. Garcia SN, Pereira-Smith O: MRGing chromatin dynamics and cellular senescence. Cell biochemistry and biophysics 2008, 50:133-141.

97. Chen M, Takano-Maruyama M, Pereira-Smith OM, Gaufo GO, Tominaga K: MRG15, a component of HAT and HDAC complexes, is essential for proliferation and differentiation of neural precursor cells. Journal of neuroscience research 2009, 87:1522-1531.

98. Adachi M, Autry AE, Covington HE, Monteggia LM: MeCP2-mediated transcription repression in the basolateral amygdala may underlie heightened anxiety in a mouse model of Rett syndrome. The Journal of neuroscience: the official journal of the Society for Neuroscience 2009, 29:4218-4227.

99. Miller Ca, Campbell SL, Sweatt JD: DNA methylation and histone acetylation work in concert to regulate memory formation and synaptic plasticity. Neurobiology of learning and memory 2008, 89:599-603.

100. Miller Ca, Sweatt JD: Covalent modification of DNA regulates memory formation. Neuron 2007, 53:857-869.

101. Poulter MO, Du L, Weaver ICG, Palkovits M, Faludi G, Merali Z, Szyf M, Anisman H: GABA(A) receptor promoter hypermethylation in suicide brain: implications for the involvement of epigenetic processes. Biological psychiatry 2008, 64:645-652.

102. Tsankova NM, Berton O, Renthal W, Kumar A, Neve RL, Nestler EJ: Sustained hippocampal chromatin regulation in a mouse model of depression and antidepressant action. Nature neuroscience 2006, 9:519-525.

103. Schneider JW, Gao Z, Li S, Farooqi M, Tang T-S, Bezprozvanny I, Frantz DE, Hsieh J: Small-molecule activation of neuronal cell fate. Nature chemical biology 2008, 4:408-410.

104. Manji HK, Moore GJ, Chen G: Clinical and preclinical evidence for the neurotrophic effects of mood stabilizers: implications for the pathophysiology and treatment of manic-depressive illness. Biological psychiatry 2000, 48:740-754.

105. Yu IT, Park J-Y, Kim SH, Lee J-S, Kim Y-S, Son H: Valproic acid promotes neuronal differentiation by induction of proneural factors in association with $\mathrm{H} 4$ acetylation. Neuropharmacology 2009, 56:473-480.

106. Semba J, Kuroda Y, Takahashi R: Potential antidepressant properties of subchronic GABA transaminase inhibitors in the forced swimming test in mice. Neuropsychobiology 1989, 21(3):152-156.

107. Schroeder Fa, Lin CL, Crusio WE, Akbarian S: Antidepressant-like effects of the histone deacetylase inhibitor, sodium butyrate, in the mouse. Biological psychiatry 2007, 62:55-64.

108. Madsen TM, Treschow a, Bengzon J, Bolwig TG, Lindvall O, Tingström a: Increased neurogenesis in a model of electroconvulsive therapy. Biological psychiatry 2000, 47:1043-1049.
109. Uchida S, Hara K, Kobayashi A, Funato H, Hobara T, Otsuki K, Yamagata H, McEwen BS, Watanabe Y: Early life stress enhances behavioral vulnerability to stress through the activation of REST4-mediated gene transcription in the medial prefrontal cortex of rodents. J Neurosci 2010, 30(45):15007-15018.

110. Bredy TW, SUn YE, Kobor MS: How the epigenome contributes to the development of psychiatric disorders. Developmental psychobiology 2010, 52:331-342.

111. Jakobsson J, Cordero MI, Bisaz R, Groner AC, Busskamp V, Bensadoun J-C, Cammas F, Losson R, Mansuy IM, Sandi C, Trono D: KAP1-mediated epigenetic repression in the forebrain modulates behavioral vulnerability to stress. Neuron $2008,60: 818-831$.

doi:10.1186/1868-7083-3-5

Cite this article as: Mateus-Pinheiro et al:. Epigenetic (de)regulation of adult hippocampal neurogenesis: implications for depression. Clinical Epigenetics 2011 3:5.

\section{Submit your next manuscript to BioMed Central and take full advantage of:}

- Convenient online submission

- Thorough peer review

- No space constraints or color figure charges

- Immediate publication on acceptance

- Inclusion in PubMed, CAS, Scopus and Google Scholar

- Research which is freely available for redistribution 